\title{
Genetic Diversity and RNA-seq Transcriptome Analysis of Tricholoma matsutake from Sichuan Province, China
}

\author{
Xiang Ding ${ }^{1,2}$, Panpan Wang², Yiling Hou*2, Mei Wang ${ }^{3}$,Wanru Hou², Yuming Li \\ 'Ecological Security and Protection Key Laboratory of Sichuan Province, Mianyang Normal University, \\ Mianyang, 621000, China \\ ${ }^{2}$ Key Laboratory of Southwest China Wildlife Resources Conservation (Ministry of Education), \\ College of Life Sciences, China West Normal University, Nanchong, 637009, China \\ ${ }^{3}$ National Center for Sweet Potato Improvement Centre of Nanchong, Nanchong Academy of Agricultural Sciences, \\ Nanchong, 637001, China
}

Received: 16 March 2016

Accepted: 19 May 2016

\begin{abstract}
The sequences of the internal transcribed spacer (ITS) regions of ectomycorrhizal fungi collected from Sichuan Province were analyzed using a PCR primer pair specific to T. matsutake. The amplified fragments were sequenced and compared with each other to build a phylogenetic tree. The mRNA deep sequencing approach was adopted to identify differentially expressed $T$. matsutake genes among the transcriptomes developed from a Xiaojin sample. A phylogenetic analysis of the aligned sequences was performed using maximum-likelihood (ML) and neighbor-joining (NJ) analyses. The results clearly showed that the KD (KM657344) and BT (KM657342) strains were more closely related to each other than to other strains. Moreover, T. matsutake from Sichuan differed from those specimens derived from Heilongjiang, Yunnam, and Guizhou provinces of China, Finland, and Japan. Furthermore, there was extremely high homology among these T. matsutake samples, despite some genetic variation. In addition, the genome of T. matsutake was sequenced using Illumina sequencing technology (RNA-seq). In all, a total of 24,549,990 reads were obtained that yielded 18,266,492 high-quality clean reads. The quality reads were excluded later. The BLAST analysis of the sequence reads against the NR database indicated that T. matsutake shared a high number of contigs with Laccaria bicolor. The results also indicated that catalytic activity, metabolic processes, metabolic pathways, and biosynthesis of secondary metabolites were the main functions identified by gene ontology (GO) and the Kyoto Encyclopedia of Genes and Genomes (KEGG) pathway analyses. Phylogenetic analysis showed that T. matsutake growing in Sichuan differed from samples growing in other regions. The differences in secondary metabolites between the Sichuan and Xiaojin samples may be due to differences in metabolic pathways. Thus the study provides a foundation for understanding T. matsutake biogeography and
\end{abstract}

*e-mail: starthlh@126.com 
origins, and identifies DEGs in the Xiaojin sample to help elucidate the molecular mechanisms in secondary metabolite synthesis.

Keywords: Tricholoma matsutake, ITS, phylogenetic, RNA-seq, differentially expressed gene, Sichuan, China

\section{Introduction}

Tricholoma matsutake (S. Ito et Imai), is one of the most valuable fungi in the world and it belongs to the genus Tricholoma (class Basidiomycota, family Tricholomaceae). T. matsutake is found in low-latitude regions of Asia, such as Sichuan, Yunnan, and Guizhou provinces of China, and in mid-latitude regions such as Japan, Korea, Finland, Canada, and Jilin Province of China [1-4]. T. matsutake usually grow in coniferous forests and broadleaf forests. In general, the range of $T$. matsutake approximately matches the distribution of coniferous genera such as Pinaceae and is less similar to the distribution of Fagaceae. Principal hosts include Pinus densiflora, Pinus thunbergii, and Pinus taiwanensis Hayata. However, Quercus aquifolioides and Quercus pannosa are the principal hosts in Sichuan Province [59]. Nonetheless, several forms of T. matsutake, such as the matsutake harvested from Xiaojin in Sichuan Province, are frequently found in mixed forests in association with Quercus. A large number of bioactive substances extracted from the fruiting bodies of T. matsutake, such as volatile compounds, polysaccharides, and polysaccharideprotein complex fractions, have been found to have immunomodulating and antioxidant properties. Despite their importance, their biogeography and origins have remained elusive, and little is known about the molecular mechanisms associated with the differences in secondary metabolite synthesis [10-14].

In recent years, molecular methods have been developed to investigate genetic variations within fungi [15-20]. Internal transcribed spacer(ITS) regions have been widely used in phylogenetic studies at different taxonomic levels because they are accessible with universal primers and their DNA sequences are variable [21-24]. Genome, transcriptome, proteome, and metabolome analyses have also played increasingly important roles in studies of the regulation of physiological activity and metabolic mechanisms. RNA-seq (transcriptome analysis) is used for transcriptome quantification and structural analysis. Transcriptome analysis lays the foundation for research into gene structure and function [25-27].

In our previous study, among several fruiting bodies from different geographical origins in southwestern China, the Xiaojin sample was found to have the best health effects and was delicious and nontoxic [28]. Furthermore, a novel heteropolysaccharide was isolated from the fruiting bodies of the Xiaojin sample [10]. Thus, the Xiaojin sample is a good model to investigate genes related to secondary metabolite synthesis. Here, the sequences of the ITS region of ectomycorrhizal fungi collected from Sichuan Province were analyzed using a specific PCR primer pair for T. matsutake. The amplified fragments were sequenced and compared with each other to build a phylogenetic tree. An mRNA deep sequencing (RNA-seq) approach was also used to identify differentially expressed genes (DEGs) in the transcriptomes of the Xiaojin sample. The goal of this study was to identify the biogeography and origins of this fungus and to identify differentially expressed genes DEGs in the Xiaojin sample to elucidate the molecular mechanisms associated with differences in secondary metabolite synthesis.

\section{Materials and Methods}

Fungal Material

T. matsutake is distributed in alpine valleys in Sichuan, located in upstream areas of the Jinsha, Yalong, and Dadu Rivers. Some samples were obtained from Jiulong, Daocheng, Yajiang, Litang, Batang, and Kangding in the Ganzi Tibetan Autonomous Prefecture, and from Rangtang, Xiaojin, and Barkam in the Aba Tibetan and Qiang Autonomous Prefecture. Other samples were obtained from Muli, Yanyuan, Yanbian, Huidong, and Mianning in the Liangshan Tibetan Autonomous Prefecture (Fig. 1). All field studies were permitted and did not involve endangered or protected species, and no samples were obtained from endangered or protected species protection zones. The names of the 37 samples are presented in Table 1 (see stock Nos. 1-37). These voucher specimens were preliminarily authenticated by Prof. Zhirong Yang

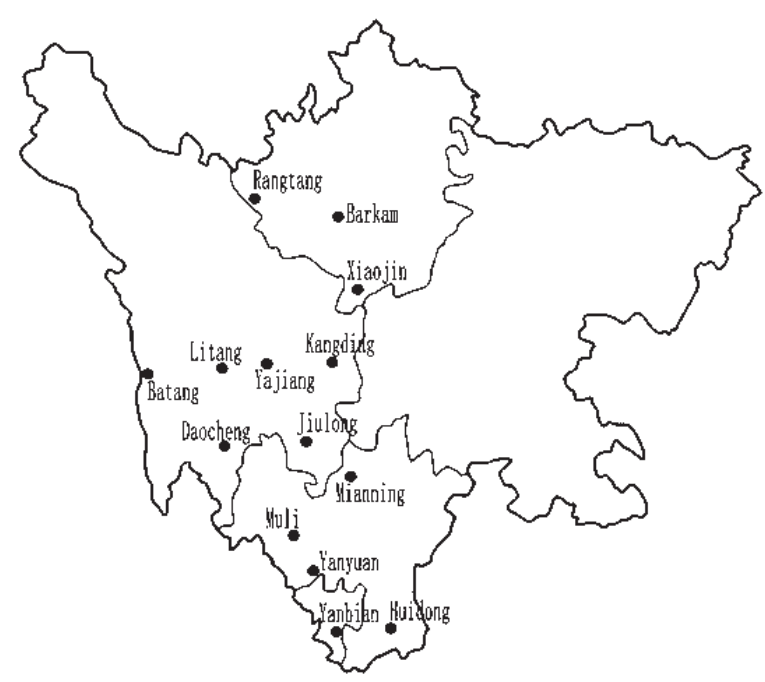

Fig. 1. Biogeographical distribution of Tricholoma matsutake varieties in Sichuan Province. 
Table 1. Tricholoma matsutake strains used in this study.

\begin{tabular}{|c|c|c|c|}
\hline $\begin{array}{c}\text { Stock } \\
\text { number }\end{array}$ & $\begin{array}{l}\text { Collection } \\
\text { number }\end{array}$ & Location & $\begin{array}{c}\text { GenBank } \\
\text { accession } \\
\text { number }\end{array}$ \\
\hline 01 & BT & Batang, Sichuan, China & KM657342 \\
\hline 02 & RT & Rangtang, Sichuan, China & KM657343 \\
\hline 03 & $\mathrm{KD}$ & Kangding, Sichuan, China & KM657344 \\
\hline 04 & YJ & Yajiang, Sichuan, China & KM657345 \\
\hline 05 & JL & Jiulong, Sichuan, China & KM657346 \\
\hline 06 & $\mathrm{DC}$ & Daocheng, Sichuan, China & KM657347 \\
\hline 07 & TLwpp & Litang, Sichuan, China & KM073272 \\
\hline 08 & TM07 & Barkam, Sichuan, China & KM102537 \\
\hline 09 & TX07 & Xiaojin, Sichuan, China & KM102538 \\
\hline 10 & YJ1-1 & Yajiang, Sichuan, China & KM581365 \\
\hline 11 & YJ2-1 & Yajiang, Sichuan, China & KM581366 \\
\hline 12 & YJ3-1 & Yajiang, Sichuan, China & KM581367 \\
\hline 13 & YJ4-1 & Yajiang, Sichuan, China & KM581368 \\
\hline 14 & YJ5-1 & Yajiang, Sichuan, China & KM581369 \\
\hline 15 & ML1-1 & Muli, Sichuan, China & KM581370 \\
\hline 16 & ML2-1 & Muli, Sichuan, China & KM581371 \\
\hline 17 & ML3-1 & Muli, Sichuan, China & KM581372 \\
\hline 18 & ML4-1 & Muli, Sichuan, China & KM581373 \\
\hline 19 & ML5-1 & Muli, Sichuan, China & KM581374 \\
\hline 20 & YY1-1 & Yanyuan, Sichuan, China & KM581375 \\
\hline 21 & YY2-1 & Yanyuan, Sichuan, China & KM581376 \\
\hline 22 & YY5-1 & Yanyuan, Sichuan, China & KM581377 \\
\hline 23 & YY5-3 & Yanyuan, Sichuan, China & KM581378 \\
\hline 24 & YB2-1 & Yanbian, Sichuan, China & KM581379 \\
\hline 25 & YB3-1 & Yanbian, Sichuan, China & KM581380 \\
\hline 26 & YB5-2 & Yanbian, Sichuan, China & KM581381 \\
\hline
\end{tabular}

(College of Life Sciences, Sichuan University, Chengdu, China) and preserved at the Key Laboratory of Southwest China Wildlife Resources Conservation (Ministry of Education), College of Life Sciences, China West Normal University.

\section{DNA Extraction}

Total genomic DNA was isolated from each sample according to the CTAB method, with slight modifications. After being triturated with liquid nitrogen, fruiting bodies of T. matsutake were transferred to $1.5 \mathrm{ml}$ microcentrifuge tubes. Subsequently, $0.5 \mathrm{ml}$ of extraction buffer was added $(100 \mathrm{mM} \mathrm{NaCl}, 200 \mathrm{mM}$ Tris- $\mathrm{HCl} \mathrm{pH}$ 8.0, $25 \mathrm{mM}$ EDTA, $0.5 \%$ SDS [w/v]). The tube was then centrifuged at $12,000 \mathrm{rpm}$ for $6 \mathrm{~min}$ after incubation at $37^{\circ} \mathrm{C}$ for $1 \mathrm{~h}$.

\begin{tabular}{|c|c|c|c|}
\hline 27 & XJ1-1 & Xiaojin, Sichuan, China & KM581382 \\
\hline 28 & XJ1-6 & Xiaojin, Sichuan, China & KM581383 \\
\hline 29 & $\mathrm{XJ} 2-2$ & Xiaojin, Sichuan, China & KM581384 \\
\hline 30 & $\mathrm{XJ} 2-5$ & Xiaojin, Sichuan, China & KM581385 \\
\hline 31 & XJ3-1 & Xiaojin, Sichuan, China & KM581386 \\
\hline 32 & HD & Huidong, Sichuan, China & KM581387 \\
\hline 33 & HD-2 & Huidong, Sichuan, China & KM581388 \\
\hline 34 & HD-4 & Huidong, Sichuan, China & KM581389 \\
\hline 35 & $\mathrm{MN}-1$ & Mianning, Sichuan, China & KM581390 \\
\hline 36 & $\mathrm{MN}-2$ & Mianning, Sichuan, China & KM581391 \\
\hline 37 & $\mathrm{MN}-3$ & Mianning, Sichuan, China & KM581392 \\
\hline 38 & j102 & Sichuan, China & EU552797 \\
\hline 39 & lt02 & Sichuan, China & EU552800 \\
\hline 40 & XJ02 & Sichuan, China & KF954507 \\
\hline 41 & YY1 & Sichuan, China & KF961006 \\
\hline 42 & YB1 & Sichuan, China & KF961007 \\
\hline 43 & ML1 & Sichuan, China & KF961009 \\
\hline 44 & MB1 & Sichuan, China & KF961010 \\
\hline 45 & HD1 & Sichuan, China & KF961011 \\
\hline 46 & TF46 & Diqing, Yunnan, China & DQ323063 \\
\hline 47 & Unknown & Guizhou, China & EU051918 \\
\hline 48 & Unknown & Heilongjiang, China & GU357477 \\
\hline 49 & Unknown & Finland & JF908736 \\
\hline 50 & Unknown & Finland & GU373503 \\
\hline 51 & Tm1 & Japan & AF204868 \\
\hline 52 & TmA-5 & Japan & AF202772 \\
\hline 53 & Tm-33 & Japan & AF204806 \\
\hline
\end{tabular}

The upper aqueous layer was moved to a new tube, and $2 \times$ CTAB buffer (100 mM Tris- $\mathrm{HCl} \mathrm{pH} 8.0,2 \%$ CTAB $[\mathrm{w} / \mathrm{v}], 1.4 \mathrm{M} \mathrm{NaCl})$ was then added. The mixture was placed in a $65^{\circ} \mathrm{C}$ water bath for $30 \mathrm{~min}$. The upper aqueous layer was extracted at least twice with an equal volume of a 1:24:25 (v:v:v) mixture of isoamyl alcohol, chloroform, and phenol. The mixture was then centrifuged at $15,000 \mathrm{rpm}$ for $12 \mathrm{~min}$, followed by extraction with isoamyl alcohol and chloroform at a ratio of 1:24 (v:v). The upper layer was transferred to a new tube, and DNA was precipitated with an equal volume of isopropanol at $-20^{\circ} \mathrm{C}$ for $10 \mathrm{~h}$. The precipitate was washed with $70 \%$ (v:v) ethanol and absolute ethanol, dried, and dissolved in TE buffer (10 mM Tris-HCl, 1 mM EDTA, pH 8.0). The concentration of the DNA was measured using a UV spectrophotometer (BIO-RAD). 
PCR Amplification and Sequencing of rDNA ITS Region

Primers were synthesized by Shanghai Invitrogen Corporation, China. The ITS region of nuclear rDNA was amplified using the primers ITS5 (5'-GGAAGTAAAAGTCGTAACAAGG-3') and ITS4 $\left(5^{\prime}\right.$-TCCTCCGCTTATTGATATGC-3') $[29,30]$. The DNA sequence amplified by this pair of primers included partial sequences of 18S rDNA and 28S rDNA and the entire sequences of ITS1, 5.8S rDNA, and ITS2. The PCR amplification was performed in a $50 \mu \mathrm{l}$ reaction volume containing $100 \mathrm{ng}$ of template DNA, $5.0 \mu \mathrm{l}$ of $10 \times$ PCR buffer $\left(\mathrm{Mg}^{2+}\right.$ free), $0.2 \mathrm{mM}$ of each dNTP, $0.2 \mu \mathrm{M}$ of each primer, $1.5 \mathrm{mM} \mathrm{MgCl}_{2}$, and $1.5 \mathrm{U}$ of Taq DNA polymerase. The PCR thermal cycling conditions were an initial denaturation at $94^{\circ} \mathrm{C}$ for $5 \mathrm{~min}$; then cycles of $40 \mathrm{~s}$ at $94^{\circ} \mathrm{C}, 30 \mathrm{~s}$ at $55^{\circ} \mathrm{C}$, and $90 \mathrm{~s}$ at $72^{\circ} \mathrm{C}$; followed by extension at $72^{\circ} \mathrm{C}$ for $5 \mathrm{~min}$. The PCR products were visualized with TAE-buffered $1.5 \%$ agarose gel electrophoresis and were then purified and sequenced by the Shanghai Invitrogen Corporation. Negative controls (no template DNA) were used in each experiment to test for the presence of nonspecific PCR products.

\section{Sequence Alignments and Analysis}

BLAST searches were conducted using the sequences of these fungi to identify homologous sequences. The DNA sequences of several strains used in this study were submitted to GenBank, and the accession numbers are shown in Table 1. Corresponding sequences from representative species were included in the phylogenetic analyses. The sequences were manually edited using DNAMAN and were aligned in Mega 5.2 using the ClustalW algorithm with the default parameters. Preliminary multiple alignments of all the sequence data were performed using ClustalX (1.8). A phylogenetic analysis of the aligned sequences was performed using the MEGA (4.0) Kimura 2-Parameter Distance Model based on ML or NJ analyses. The NJ and ML analyses included bootstrapping using 1,000 replicates.

\section{Basic Statistics of the Quality of the Original Sequencing Reads}

Transcriptome sequencing was conducted at Beijing GGT Co., Ltd. RNA Solexa sequencing yielded the sample data. Image data were transformed into sequence data by base calling. The data were provided in FASTQ format, including the names of the sequencing reads, sequences, and quality measures. Each FASTQ-formatted file is described by four lines: the first and third rows are the sequence names generated by the sequencing, the second line is the sequence, and the fourth line is the sequence quality.

\section{Quality Pretreatment of Reads}

Linker sequences, low-quality reads, and contaminating reads were removed from the original data to obtain clean sequence data. The length distribution statistics were then computed as follows:

1. Remove low-quality reads: quality threshold 20 (error rate $=1 \%$ ), proportional threshold $=40 \%$.

2. Remove reads with proportion of the larger sequence of $\mathrm{N}$; threshold $=4 \%$.

3. Remove the linker sequence.

4. Compute the length distribution statistics of the clean sequence.

\section{Assembly Analysis}

Trinity is a novel method for the efficient and robust de novo reconstruction of transcriptomes from RNAseq data. Trinity combines three independent software modules: Inchworm, Chrysalis, and Butterfly, which are sequentially applied to process large volumes of RNA-seq reads. Trinity partitions the sequence data into many individual de Bruijn graphs (each representing the transcriptional complexity at a given gene or locus) and then processes each graph independently to extract fulllength splicing isoforms to tease apart transcripts derived from paralogous genes. Briefly, the process consists of the following:

1. Inchworm assembles the RNA-seq data into the unique sequences of transcripts, often generating full-length transcripts for a dominant isoform, but then reports only the unique portions of alternatively spliced transcripts.

2. Chrysalis clusters the Inchworm contigs into clusters and constructs complete de Bruijn graphs for each cluster. Each cluster represents the full transcriptional complexity for a given gene (or sets of genes that share sequences in common). Chrysalis then partitions the full read set among these disjointed graphs.

3. Butterfly then processes the individual graphs in parallel, tracing the paths that read and pairs of reads taken within the graph, ultimately reporting fulllength transcripts for alternatively spliced isoforms, and teasing apart transcripts that correspond to paralogous genes.

\section{Trinity Results and Common Database Comparison}

BLASTX was performed against the COG, EST, NR, Pfam, UniProt, and other protein databases to identify all the unigenes, The results, which provided the corresponding database and degree of homology standard (E-value), were used to functionally annotate the genes. The annotation returned a value of NULL when no homologous genes were identified in the database. 


\section{GO Annotation and KEGG Enrichment Analyses}

All genes were annotated for protein function using InterProScan (www.ebi.ac.uk/interpro) and BLASTX against the NCBI NR database. The resulting InterPro and BLAST annotations were converted into GO annotations, and all GO terms were mapped to GO slim categories. The significance of the functional GO slim enrichment was evaluated using Fisher's exact test within Blast2GO with a false discovery rate (FDR) of $<0.05$. Significantly enriched KEGG pathways were identified with KOBAS 2.0 using a hypergeometric test and the Benjamini-Hochberg FDR correction.

\section{Results and Discussion}

\section{Phylogenetic Analyses Based on the Sequence of the ITS Region}

A part of the ITS region of T. matsutake samples of various geographical origins was amplified using PCR and a T. matsutake-specific primer pair. BLAST searches were conducted based on the sequences of 37 T. matsutake samples from Sichuan Province $\left(486,000 \mathrm{~km}^{2}\right)$ to identify homologous sequences (Fig. 1). The ITS of the fungus was sequenced and submitted to GenBank (Table 1). A phylogenetic tree of aligned sequences was constructed using maximum-likelihood (ML) (Figs 2 and 3) and neighbor-joining (NJ) analyses (Figs 4 and 5). Both trees clearly showed two phylogenetic lineages arising from the root. The results showed differences between the ML tree and the $\mathrm{NJ}$ tree.

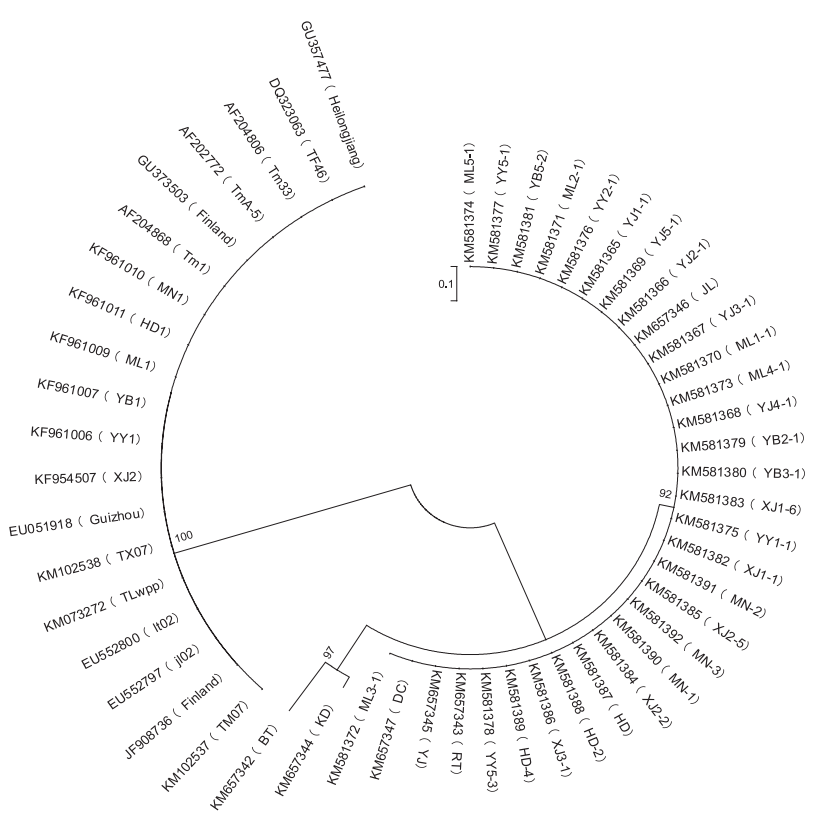

Fig. 2. Topology of Tricholoma matsutake obtained using the maximum-likelihood (ML) method.
The first clade at the top of these two trees consisted of 34 individuals of T. matsutake, but strains KD (KM657344) and BT (KM657342) were shown to be more closely related to each other than to other strains. The T. Matsutake samples from the three regions of Sichuan (shown in Fig. 1) had a closer genetic relationship in adjacent regions. For example, samples from Jiulong, Daocheng, Yajiang, Litang, Batang, and Kangding in the Ganzi Tibetan Autonomous Prefecture had a close genetic relationship, whereas samples from Rangtang, Xiaojin, and Barkam of the Aba Tibetan and Qiang Autonomous Prefecture had a closer genetic relationship. However, the

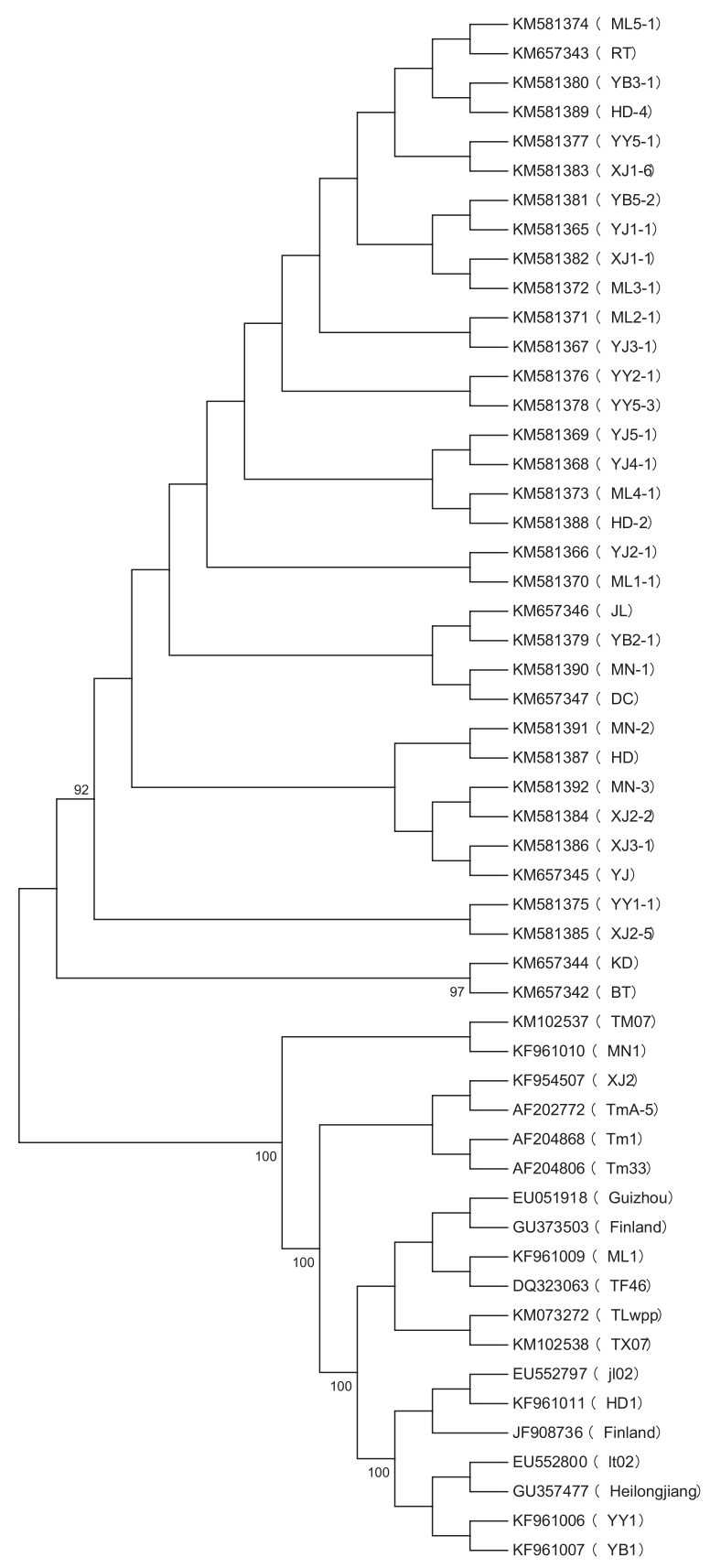

Fig. 3. Maximum-likelihood (ML) tree generated for Tricholoma matsutake obtained by the ML search. The number below each internode represents the percentage of 1,000 bootstrap replicates supporting binary partition. 


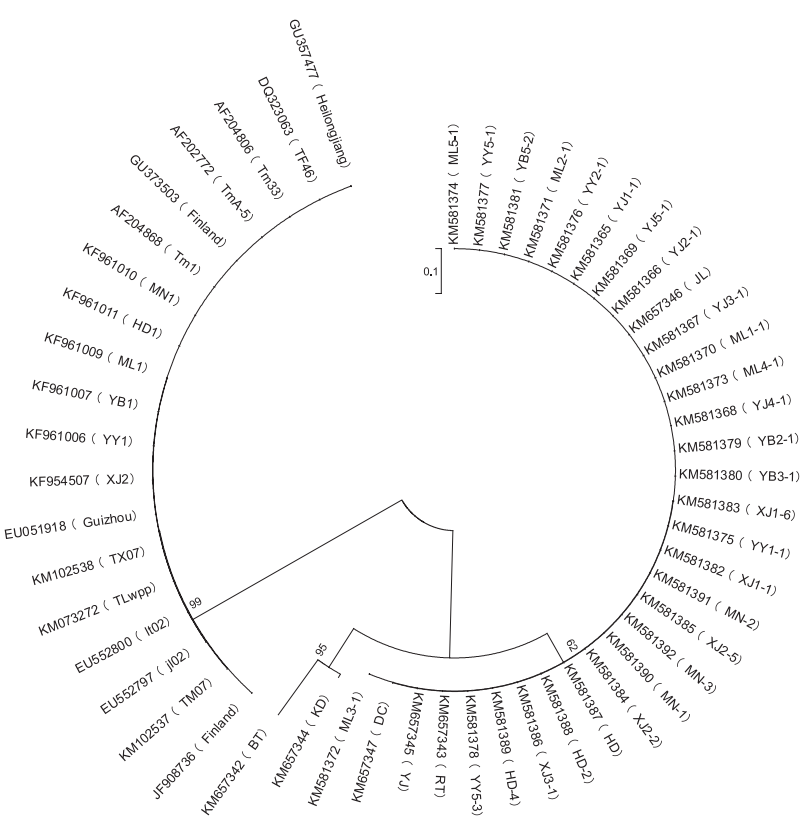

Fig. 4. Topology of Tricholoma matsutake obtained using the neighbor-joining (NJ) method.

relationship between T. matsutake from the Sichuan area and samples from other places was distant because the first clade at the top of these two trees consisted of 34 samples of T. matsutake that were all from Sichuan Province.

The second clade at the bottom of the tree consisted of 19 strains, all of which were closely related, including samples from the Heilongjiang, Yunnan, Guizhou, Finland, and Japan. T. matsutake from northeastern China is known as Pinus densiflora, whereas T. matsutake from Japan is mainly known as Pinus thunbergii Parl. and Pinus densiflora. However, Quercus aquifolioides and $Q$. pannosa are the major host plants for Sichuan $T$. matsutake. Notably, TM07 (KM102537) from Barkam (Sichuan, China) was distinct in the NJ trees. Analyses of both lineages indicated that the genetic relationship among samples KM102537, KM102538, and KM073272 was closer than that between other samples from Sichuan Province, which are in the second clade at the bottom of these two trees. Overall, individuals from Sichuan showed a high level of homogeneity. However, T. matsutake from Sichuan differed from samples from Heilongjiang, Yunnan, and Guizhou provinces of China, plus Finland and Japan. It was concluded that the homology among these T. matsutake samples was extremely high, despite some genetic variation.

\section{Analysis of the Transcriptome Database}

Based on the merits of transcriptome analyses, $T$. matsutake samples from Xiaojin were subjected to RNAseq on the Illumina sequencing platform. The quality of the original read data from the samples was good, and the base quality exceeded 32 in all cases, which fulfilled the requirements for follow-up analysis (Fig. 6). A total of $24,549,990$ reads were obtained by sequencing, and

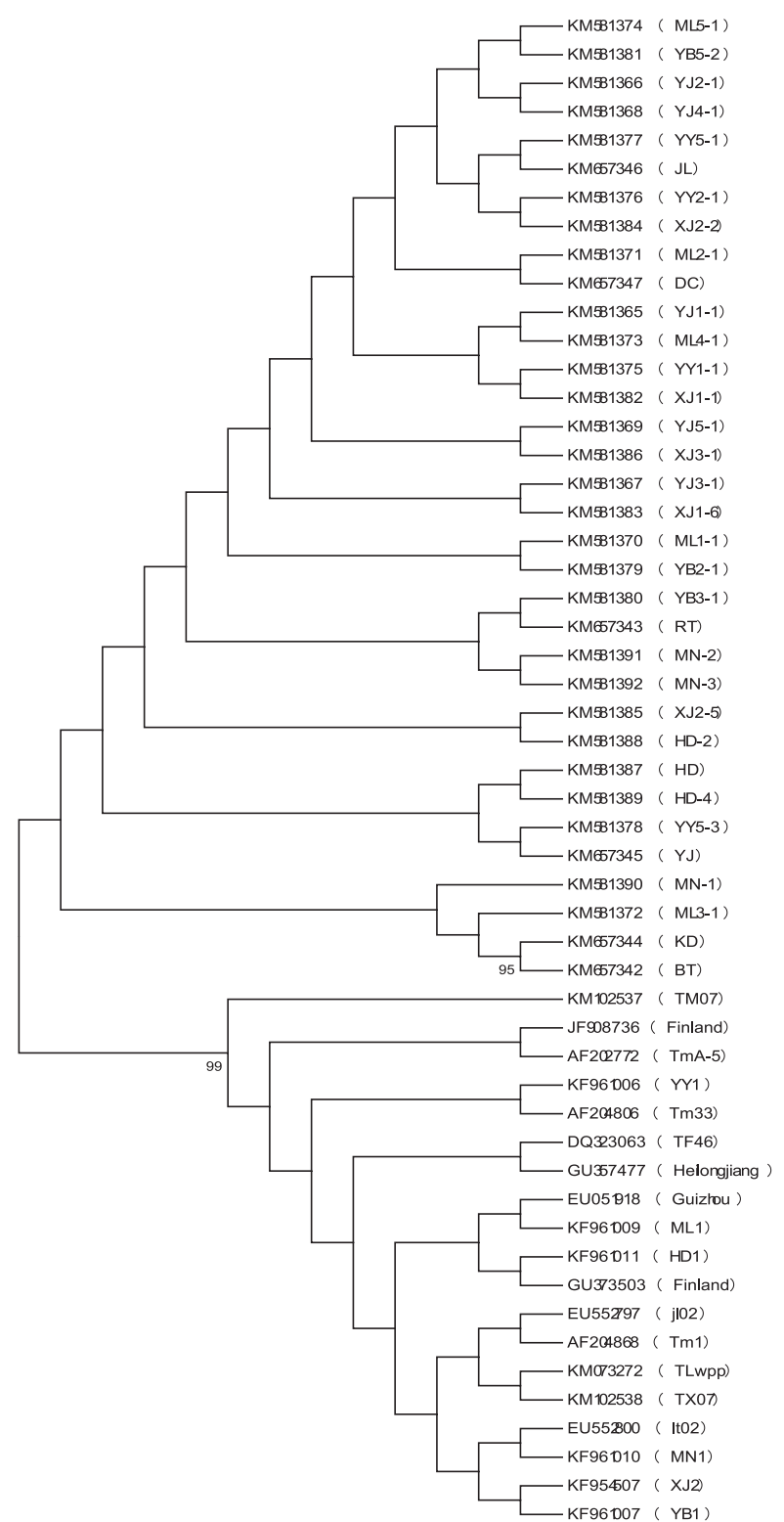

Fig. 5. Neighbor-joining (NJ) tree generated for Tricholoma matsutake obtained by the NJ search. The number below each internode represents the percentage of 1,000 bootstrap replicates supporting that binary partition.

$18,266,492$ high-quality and clean reads were selected after low-quality reads were excluded (Table 2). Trinity software generated clean data, and 32,098 contigs were obtained by assembling the reads to yield an N50 contig size of 812 bp (i.e., $50 \%$ of the genomic fragments were this length or longer; Table 3). There were 8,909 contigs $(27.82 \%)$ with lengths between 201 and 300 bp. Moreover, 5,185, 3,677, 2,752, 2,114, 1,734, 1,362, $1,147,1,625,1,180$, and 2,336 contigs had lengths of 301-400 bp, 401-500 bp, 501-600 bp, 601-700 bp, 701-800 bp, 801-900 bp, 901-1,000 bp, 1,001-1,200 bp, 1,201-1,400 bp, and greater than 1,400 bp, respectively (Table 4, Figs 7-8). Furthermore, 26,729 unigenes were obtained by assembling the scaffolds, and the proportions of A, T, C, and G were almost equal (Table 5). 


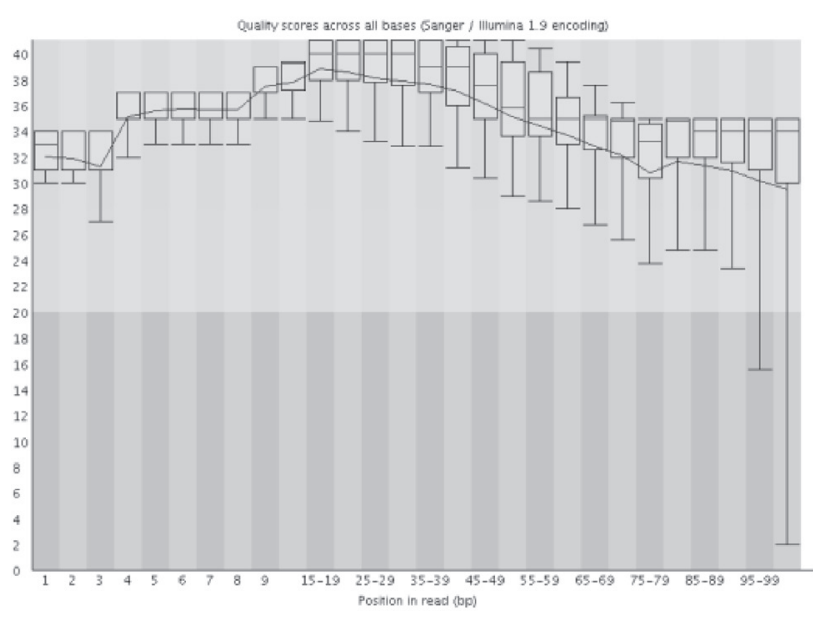

Fig. 6. Boxplot graph of sequence quality of Xiaojin Tricholoma matsutake genome.

\section{Functional Annotation and Classification}

Based on a comparison of the unigenes with the COG, EST, non-redundant (NR), Pfam, and UniProt databases, genome annotations and E-values were obtained. The results showed that there were 9,487, 6,856, 21,482, 18,537 , and 12,715 matches in the COG, EST, NR, Pfam, and UniProt databases, respectively. The Venn diagram is shown in Fig. 9 and Table 6.

The identified unigenes with BLASTX hits in the NR database showed the highest homology to Laccaria bicolor S238N-H82, with a contig number of 6,559 $(30.33 \%)$. Furthermore, 1,647, 1,540, and 1,018 contigs were obtained with Coprinopsis cinerea okayama 7\#130, Serpula lacrymans var. lacrymans S7.9 and Pseudomonas fluorescens WH6, respectively (Table 7).

Moreover, the BLAST analysis against the NR database indicated that T. matsutake shared a high number of contigs with Laccaria bicolor. L. bicolor grows in mixed broadleaf conifer forests in Sichuan, the Tibet Autonomous Region of Southern China, and Yunnan Province. The similarity to the host of T. matsutake might be responsible for the high contig number.

\section{GO and KEGG Analyses}

The Gene Ontology (GO) Consortium categorizes genes based on molecular function, cellular component, and biological process.

All the unigenes in the transcriptome of T. matsutake were examined for GO functional prediction and
Table 3. Features of the Xiaojin Tricholoma matsutake genome.

\begin{tabular}{|c|c|c|}
\hline Item & Contig length & Unique gene length \\
\hline N75 & 458 & 418 \\
\hline N50 & 813 & 741 \\
\hline N25 & 1,334 & 1,248 \\
\hline Minimum & 201 & 201 \\
\hline Maximum & 5,879 & 5,879 \\
\hline Average & 620 & 578 \\
\hline Count & 32,098 & 26,729 \\
\hline $\begin{array}{c}\text { Total } \\
\text { length }\end{array}$ & $1,992,214$ & $15,469,839$ \\
\hline
\end{tabular}

Table 4. Length distribution of assembled contigs and unigenes of Xiaojin Tricholoma matsutake genome.

\begin{tabular}{|c|c|c|}
\hline $\begin{array}{l}\text { Nucleotide length } \\
\text { (bp) }\end{array}$ & $\begin{array}{l}\text { Contig } \\
\text { number }\end{array}$ & $\begin{array}{l}\text { Unique } \\
\text { genes }\end{array}$ \\
\hline $75-100$ & 0 & 0 \\
\hline $101-200$ & 0 & 0 \\
\hline $201-300$ & 8,909 & 8,183 \\
\hline $301-400$ & 5,185 & 4,577 \\
\hline $401-500$ & 3,677 & 3,139 \\
\hline $501-600$ & 2,752 & 2,237 \\
\hline $601-700$ & 2,114 & 1,652 \\
\hline $701-800$ & 1,734 & 1,354 \\
\hline $801-900$ & 1,362 & 1,034 \\
\hline $901-1,000$ & 1,147 & 852 \\
\hline $1,001-1,200$ & 1,625 & 1,191 \\
\hline $1,201-1,400$ & 1,180 & 841 \\
\hline $1,401-1,600$ & 714 & 524 \\
\hline $1,601-1,800$ & 500 & 346 \\
\hline $1,801-2,000$ & 322 & 233 \\
\hline $2,001-2,500$ & 486 & 301 \\
\hline $2501-3000$ & 195 & 104 \\
\hline$>3,000$ & 119 & 88 \\
\hline
\end{tabular}

classification. All the unigenes were broadly divided into 55 categories according to their function. The largest set

Table 2. RNA-seq statistical data of Xiaojin Tricholoma matsutake.

\begin{tabular}{|c|c|c|c|c|c|}
\hline \multirow{2}{*}{ Sample } & \multicolumn{2}{|c|}{ Raw Data } & \multicolumn{2}{c|}{ Valid Data } & Valid \\
\cline { 2 - 5 } & Read & Length & Read & Length & Ratio \\
\hline LCF-song & $24,549,990$ & 100 & $18,266,492$ & 90.03972 & $74.41 \%$ \\
\hline
\end{tabular}




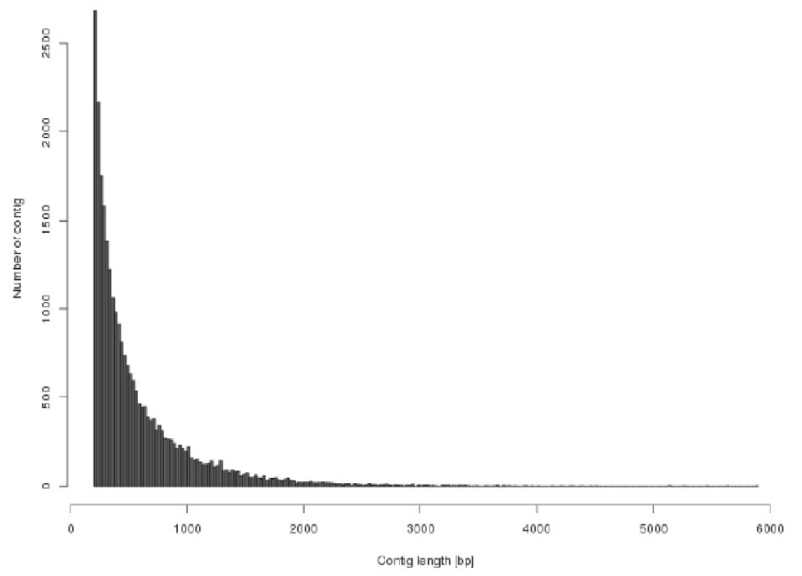

Fig. 7. Histogram of length distribution of assembled contigs derived from the Xiaojin Tricholoma matsutake genome.

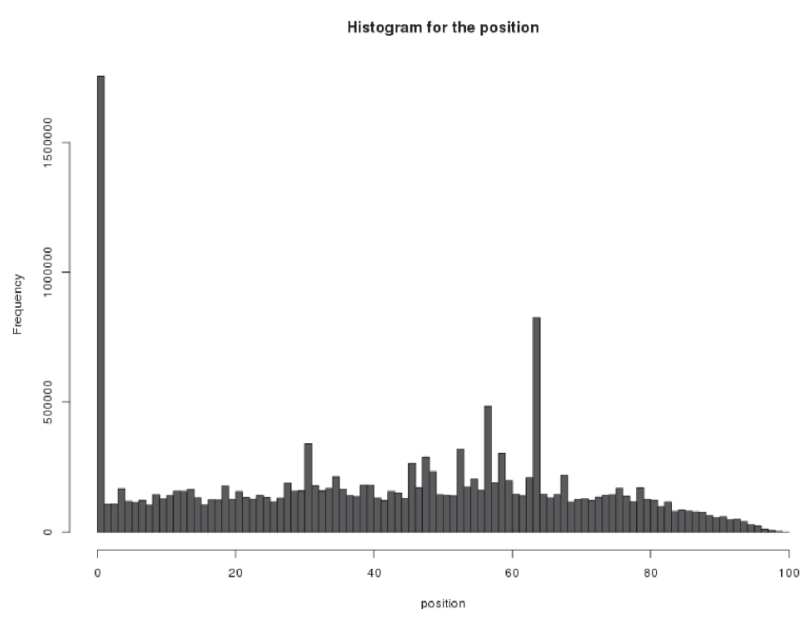

Fig. 8. Results of Kolmogorov-Smirnov test on genome of Xiaojin Tricholoma matsutake.

was assigned to the cluster "catalytic activity" $(22,304$; $19.96 \%$ of total unigenes), followed by "metabolic process" (20,093; 17.98\%), "binding" $(17,897 ; 16.02 \%)$, "cell part" (7478; 6.69\%), “organelle" (7031; 6.29\%), "regulation of biological process" (3941; 3.53\%), "membrane part" (3698; 3.31\%), "cellular process" (3663; 3.28\%), and "establishment of localization" (3537; 3.17\%) (Table 8 and Fig. 10).

Table 5. Nucleotide data of Xiaojin Tricholoma matsutake genome.

\begin{tabular}{|c|c|c|}
\hline Nucleotide & Count & Frequency \\
\hline Adenine (A) & $4,875,123$ & $24.43 \%$ \\
\hline Cytosine (C) & $5,106,053$ & $25.59 \%$ \\
\hline Guanine (G) & $5,101,894$ & $25.57 \%$ \\
\hline Thymine (T) & $4,839,077$ & $24.25 \%$ \\
\hline Any nucleotide $(\mathrm{N})$ & 32,098 & $0.16 \%$ \\
\hline
\end{tabular}

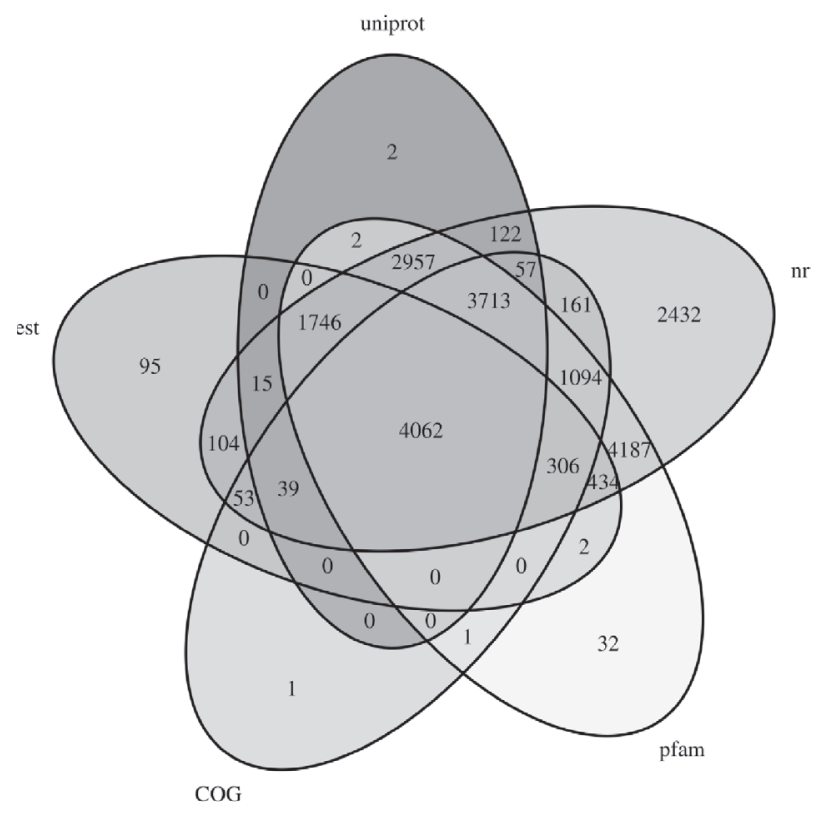

Fig. 9. Venn diagram of Trinity BLAST comparison of genome of Xiaojin Tricholoma matsutake with other databases.

Table 6. Trinity BLAST comparison of genome of Xiaojin Tricholoma matsutake with other databases.

\begin{tabular}{|c|c|c|}
\hline Sample name & Database type & Match number \\
\hline LCF-song & COG & 9,487 \\
\hline LCF-song & EST & 6,856 \\
\hline LCF-song & NR & 21,482 \\
\hline LCF-song & Pfam & 18,536 \\
\hline LCF-song & UniProt & 12,715 \\
\hline
\end{tabular}

Table 7. Species homology analysis of Xiaojin Tricholoma matsutake and other species.

\begin{tabular}{|c|c|}
\hline Species & $\begin{array}{c}\text { Contig } \\
\text { number }\end{array}$ \\
\hline Laccaria bicolor S238N-H82 & 6,559 \\
\hline Coprinopsis cinerea okayama $7 \# 130$ & 1,647 \\
\hline Serpula lacrymans var. lacrymans S7.9 & 1,540 \\
\hline Pseudomonas fluorescens WH6 & 1,018 \\
\hline Pseudomonas fluorescens SBW25 & 837 \\
\hline Schizophyllum commune H4-8 & 721 \\
\hline Pseudomonas extremaustralis $14-3$ substr. & 718 \\
\hline 14-3b & 536 \\
\hline Moniliophthora perniciosa FA553 & 528 \\
\hline Serpula lacrymans var. lacrymans S7.3 & 514 \\
\hline Fibroporia radiculosa & 6,855 \\
\hline Other &
\end{tabular}


Table 8. Gene ontology functional classification of Xiaojin Tricholoma matsutake genome (Count $>1,000)$.

\begin{tabular}{|c|c|c|}
\hline Go_term & Count & Description \\
\hline GO:0003824 & 22,304 & catalytic activity \\
\hline GO:0005215 & 1,820 & transporter activity \\
\hline GO:0005488 & 17,897 & binding \\
\hline GO:0008152 & 20,093 & metabolic process \\
\hline GO:0009987 & 3,663 & cellular process \\
\hline GO:0016020 & 2,934 & membrane \\
\hline GO:0032991 & 3,126 & macromolecular complex \\
\hline GO:0043226 & 7,031 & organelle \\
\hline GO:0044422 & 1,137 & organelle part \\
\hline GO:0044425 & 3,698 & membrane part \\
\hline GO:0044464 & 7,478 & cell part \\
\hline GO:0044699 & 2,628 & single-organism process \\
\hline GO:0050789 & 3,941 & $\begin{array}{c}\text { regulation of biological } \\
\text { process }\end{array}$ \\
\hline GO:0050896 & 2,385 & response to stimulus \\
\hline GO:0051234 & 3,537 & $\begin{array}{l}\text { establishment of } \\
\text { localization }\end{array}$ \\
\hline GO:0071840 & 2,096 & $\begin{array}{c}\text { cellular component } \\
\text { organization or biogenesis }\end{array}$ \\
\hline
\end{tabular}

The active biological pathways in $T$. matsutake were mapped to the annotated sequences by reference to pathways in KEGG. The unigenes were divided into 190 pathways. The largest set was "metabolic pathways" $(812 ; 16.75 \%)$, followed by "biosynthesis of secondary metabolites" (505; 10.42\%), "microbial metabolism" (451; 9.30\%), and "ABC transporters" (192; 3.96\%). In

Table 9. KEGG analysis of Xiaojin Tricholoma matsutake genome (Count $>100)$.

\begin{tabular}{|c|c|c|}
\hline Pathway & Count & Description \\
\hline map00970 & 125 & $\begin{array}{c}\text { Aminoacyl-tRNA } \\
\text { biosynthesis }\end{array}$ \\
\hline map01100 & 812 & Metabolic pathways \\
\hline map01110 & 505 & $\begin{array}{c}\text { Biosynthesis of secondary } \\
\text { metabolites }\end{array}$ \\
\hline map01120 & 451 & $\begin{array}{c}\text { Microbial metabolism in } \\
\text { diverse environments }\end{array}$ \\
\hline map02010 & 192 & ABC transporters \\
\hline map02020 & 143 & Two-component system \\
\hline map03010 & 126 & Ribosome \\
\hline map03018 & 125 & RNA degradation \\
\hline map03040 & 142 & Spliceosome \\
\hline
\end{tabular}

our previous study, organic solvent extraction-distillation followed by GC-MS combined with quantification by peak area normalization was used to analyze volatile compounds in fresh fruiting bodies of $T$. matsutake collected from different geographical origins in southwestern China. Twenty-seven volatile compounds were identified from 66 species, including seven alcohols, three carbonyls, 11 acids and esters, three terpene hydrocarbons, and three miscellaneous components. The volatile compounds in the Xiaojin sample differed from those in T. matsutake collected from different geographical origins in southwestern China, and the Xiaojin sample had the best health effects and was delicious and nontoxic. Additionally, a novel heteropolysaccharide (TMP-A) was isolated from the fruiting bodies of $T$. matsutake from Xiaojin County. TMP-A has a backbone of 1,4- $\beta$-glucopyranose that branches at O-6 into an $(1 \rightarrow 3)$ - $\alpha$-galactopyranose residue and terminates at an $\alpha$-xylopyranose residue. The difference in the secondary metabolites from the Xiaojin sample may be due to differences in metabolic pathways.

Many pathways are involved in fungal growth, development, differentiation, and death. Three signaling pathways were identified in T. matsutake: the MAPK, PI3K, and Notch pathways. A total of 41 unigenes were identified. Moreover, 9 and 32 genes were involved in the MAPK signaling pathway (Map ID: 04010) and the yeast MAPK signaling pathway (Map ID: 04011), respectively. Three genes and one gene were involved in the PI3K-Akt (Map ID: 04151) and Notch signaling pathways (Map ID: 04330). Notably, two tumor-related pathways were identified in T. matsutake: p53 (Map ID: 04115) and Wnt (Map ID: 04310), plus xamples of alanine, aspartate, and glutamate metabolism (Map ID: 00250) (Table 9 and Fig. 11).

The analysis of the $T$. matsutake transcriptome provided information for further research to elucidate the physiology and medicinal value of this fungus.

\section{Conclusion}

This study analyzed the homology of T. matsutake based on ITS sequences, and the results were congruent with those of previous studies. In total, T. matsutake samples from Sichuan showed a high level of homology. However, the T. matsutake samples from Sichuan differed from samples from Heilongjiang, Yunnan, and Guizhou provinces of China, and Finland and Japan. T. matsutake from northeastern China is known as Pinus densiflora, whereas T. matsutake from Japan is mainly known as Pinus thunbergii Parl. and Pinus densiflora. However, Quercus aquifolioides and $Q$. pannosa are the major host plants for T. matsutake in Sichuan. Moreover, a BLAST analysis against the NR database indicated that $T$. matsutake shared a high number of contigs with Laccaria bicolor. L. bicolor grows in mixed broadleaf conifer forests in Sichuan, the Tibet Autonomous Region of Southern China, and Yunnan. The similarity in hosts between T. matsutake 


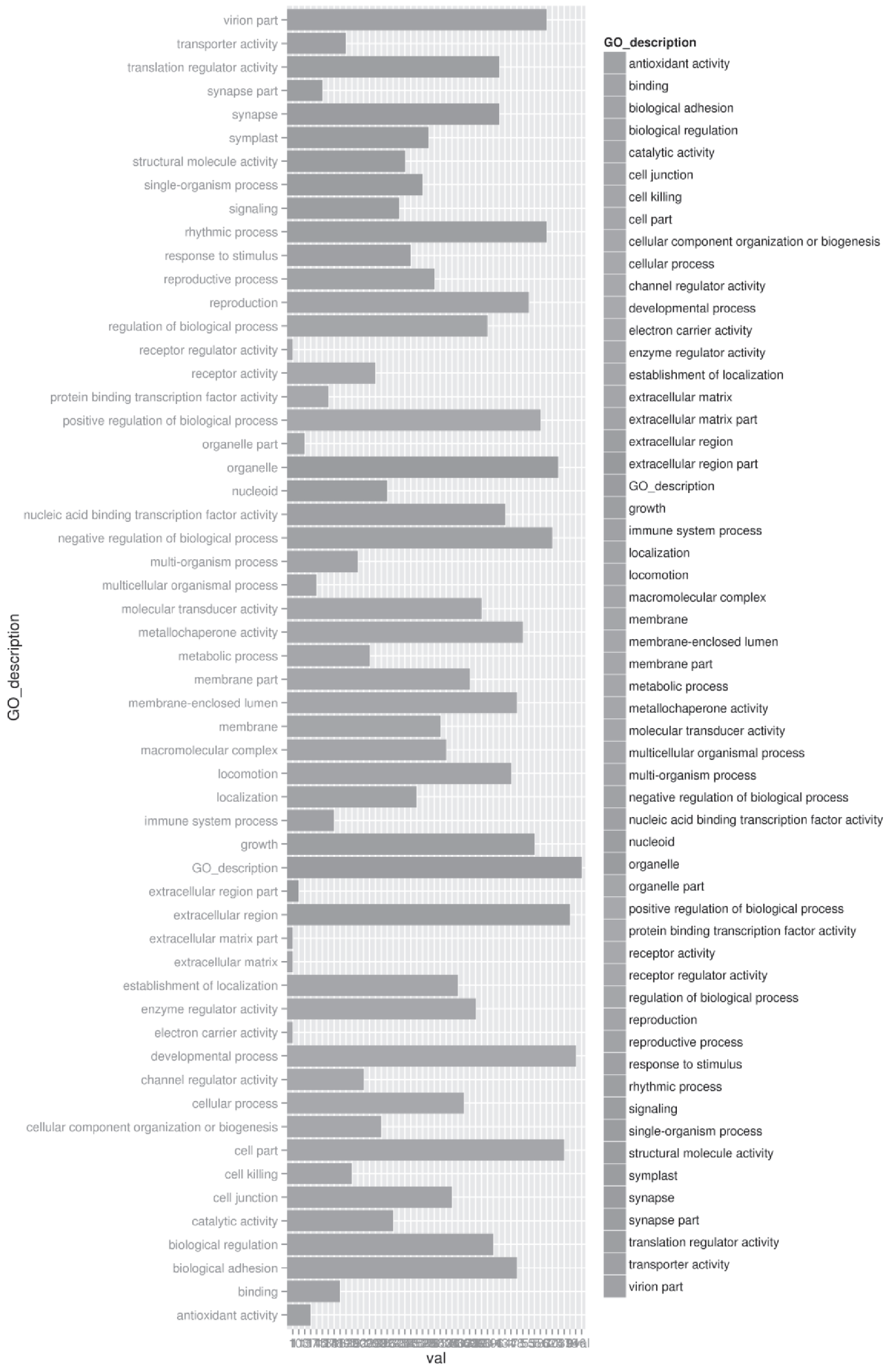

Fig. 10. GO analysis of Xiaojin Tricholoma matsutake genome.

and L. bicolor may be partly responsible for the high contig number. Notably, the influence of differences in host plants on genetic differentiation among different $T$. matsutake individuals remains to be fully explored. In the present study, we also used next-generation sequencing technology for the de novo assembly of the transcriptome of T. matsutake from Xiaojin. The transcriptome analysis of Xiaojin T. matsutake provided information for further research to elucidate the physiology and medicinal value of this fungus.

\section{Abbreviations}

RNA-seq: RNA sequencing GO: Gene ontology 
ALAFINE, ASPARTATE AND GLUTAMATE METABOLISM

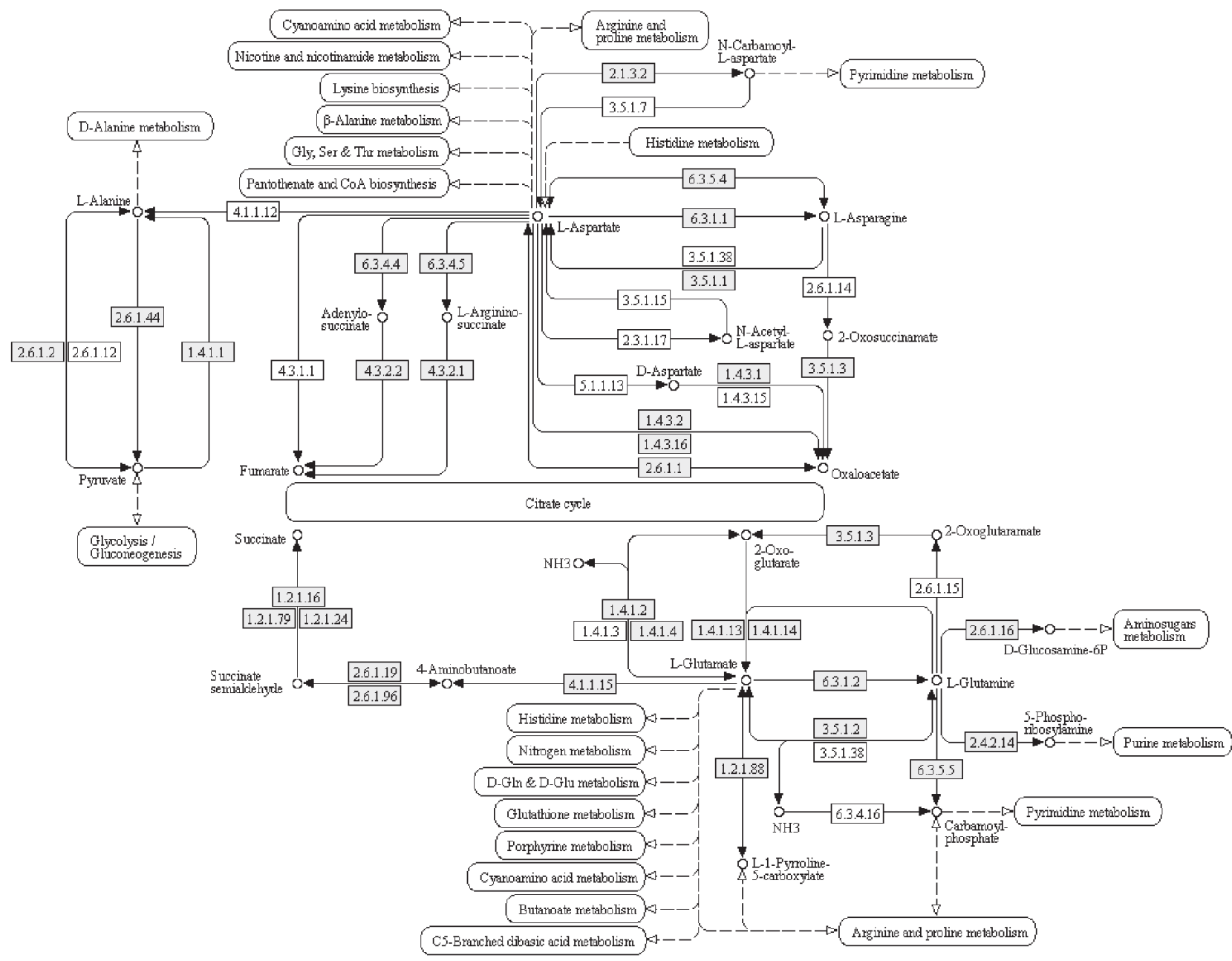

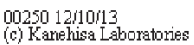

Fig. 11. KEGG analysis of Xiaojin Tricholoma matsutake genome. Core genes are colored gray; uncolored genes do not exist in the genome.

KEGG: Kyoto Encyclopedia of Genes and Genomes BLAST: Basic local alignment search tool DEG: Differentially expressed gene

\section{Authors' Contributions}

$\mathrm{XD}$ and $\mathrm{YH}$ designed the project. XD and MW performed the sample collection and RNA isolation. XD, $\mathrm{YH}, \mathrm{WH}, \mathrm{YL}$, and $\mathrm{PW}$ performed the bioinformatics analysis. $\mathrm{XD}$ and $\mathrm{YH}$ drafted the manuscript, and $\mathrm{YH}$ edited it. All the authors read and approved the final manuscript.

\section{Acknowledgements}

This project was supported by the National Natural S cience Foundation of China (31400016 and 31200012), the Key Laboratory Fund Project of Ecological security and protection of Sichuan Province (ESP201307 and ESP1408), the Application Foundation Project of Sichua n Province (2013JY0094), the Science and Technology S upport Project of Sichuan Province (2014SZ0020 and 201 4FZ0024), the Cultivate Major Projects of Sichuan Provi nce (14CZ0016 and 16CZ0018), and the Open Foundation of Microbial Resources and Drug Development of Key Laboratory and of Guizhou Province (GZMRD-2014-00 2).

\section{References}

1. BAO D., KOIKE A., YAO F., YAMANAKA K., AIMI T., KITAMOTO Y. Analyses of the genetic diversity of matsutake isolates collected from different ecological environments in Asia. J. Wood. Sci. 53 (4), 344, 2007.

2. BWEGIUS N., DANELL E. The Swedish matsutake (Tricholoma nauseosum syn. T. matsutake): distribution, 
abundance and ecology. Scan. J. Forest. Res. 15 3), 318, 2000.

3. SHA T., ZHANG H., DING H., LI Z., CHENG L., ZHAO Z., ZHANG Y. Genetic diversity of Tricholoma matsutake in Yunnan Province. Chin. Sci. Bull. 52 (9), 1212, 2007.

4. YANG X., HE J., LI C., MA J., YANG Y., XU J. Matsutake trade in Yunnan Province, China: an overview. Econ. Bot. 62 (3), 269. 2008.

5. GILL W.M., GUERIN-LAGUETTE A., LAPEYRIE F., SUZUKI K. Matsutake - morphological evidence of ectomycorrhiza formation between Tricholoma matsutake and host roots in a pure Pinus densiflora forest stand. New. Phytol. 147 (147), 381, 2000.

6. VAARIO L., HEINONSALO J., SPETZ P., PENNANEN T., HEINONEN J., TERVAHAUTA A., FRITZE H. The ectomycorrhizal fungus Tricholoma matsutake is a facultative saprotroph in vitro. Mycorrhiza. 22 (6), 409, 2012.

7. KUSUDA M., NAGAI M., HUR T., UEDA M., TERASHITA T. Purification and some properties of $\alpha$-amylase from an ectomycorrhizal fungus, Tricholoma matsutake. Mycoscience. 44 (4), 311, 2003.

8. VISNOVSKY S.B., CUMMINGS N., GUERINLAGUETTE A., WANG Y., YAMADA A., KOBAYASHI H., KAWAI M., PITMAN A.R. Detection of the edible ectomycorrhizal fungus Lyophyllum shimeji colonising seedlings of cultivated conifer species in New Zealand. Mycorrhiza 24 (6), 453, 2014.

9. KATAOKA R., SIDDIQUI Z. A., KIKUCHI J., ANDO M., SRIWATI R., NOZAKI A., FUTAI K. Detecting nonculturable bacteria in the active mycorrhizal zone of the pine mushroom Tricholoma matsutake. J. Microbiol. 50 (2), 199, 2012.

10. XIANG D., YILING H., WANRU H. Structure feature and antitumor activity of a novel polysaccharide isolated from Lactarius deliciosus Gray, Carbohyd. Polym. 89 (2), 397, 2012.

11. XIANG D., YILING H., YUANXIU Z., PANPAN W., LEI F., HONGQING Z., NAN Z., HANG Q., WEI Q., FANG W., WANRU H. Structure elucidation, anticancer and antioxidant activities of a novel polysaccharide from Gomphus clavatus Gray, Oncol. Rep. 33 (6), 3162, 2015.

12. FANG W., YILING H., XIANG D., WANRU H., BO S., TING W., JIAN L., YICHUN Z. Structure elucidation and antioxidant effect of a novel polysaccharide from Lactarius camphoratum (Bull.) Fr., Int. J. Biol. Macromol. 62 (10), 131, 2013.

13. XIANG D., YILING H., WANRU H. Structure elucidation and antioxidant activity of a novel polysaccharide isolated from Boletus speciosus Forst, Int. J. Biol. Macromol. 50 (3), 613, 2012.

14. DING X., FENG S., CAO M., LI M., TANG J., GUO C., JIE Z., QUN S., ZHIRONG Y., JIAN Z. Structure characterization of polysaccharide isolated from the fruiting bodies of Tricholoma matsutake. Carbohydr. Polym. 81 (4), 942, 2010.

15. GUERIN-LAGUETTE A., MATSUSHITA N., KIKUCHI K., IWASE K., LAPEYRIE F., SUZUKI K. Identification of a prevalent Tricholoma matsutake ribotype in Japan by rDNA IGS1 spacer characterization. Mycol. Res. 106 (2), 435, 2002.

16. LIAN C., HOGETSU T., MATSUSHITA N., GUERINLAGUETTE A., SUZUKI K., YAMADA A. Development of microsatellite markers from an ectomycorrhizal fungus,
Tricholoma matsutake, by an ISSR-suppression-PCR method. Mycorrhiza. 13 (1), 27, 2003.

17. MURATA H., BABASAKI K., SAEGUSA T., TAKEMOTO K., YAMADAA., OHTAA. Traceability of Asian Matsutake, specialty mushrooms produced by the ectomycorrhizal basidiomycete Tricholoma matsutake, on the basis of retroelement-based DNA markers. Appl. Environ. Microbiol. 74 (7), 2023, 2008.

18. XU J., CADORIN M., LIANG Y., YANG Z.L. DNA-based geographic typing of the gourmet mushroom Tricholoma matsutake traded in China. Mycoscience. 51 (3), 248, 2010.

19. HWANG S., KIM J. Secondary structural and phylogenetic implications of nuclear large subunit ribosomal RNA in the ectomycorrhizal fungus Tricholoma matsutake. Curr. Microbio. 40 (4), 250, 2000.

20. MATSUSHITA N., KIKUCHI K., SASAKI Y., GUERINLAGUETTE A., LAPEYRIE F., VAARIO L., SUZUKI K. Genetic relationship of Tricholoma matsutake and T.nauseosum from the Northern hemisphere based on analyses of ribosomal DNA spacer regions. Mycoscience. 46 (2), 90, 2005.

21. CHAPELA I.H., GARBELOTTO M. Phylogeography and evolution in matsutake and close allies inferred by analyses of ITS sequences and AFLPs. Mycologia. 96 (4), 730, 2004.

22. DUNSTAN W.A., DELL B., MALAJCZUK N., IWASE K. Detection of the ectomycorrhizal fungus Tricholoma matsutake and some related species with specific ITS primers. Mycoscience. 41 (1), 33, 2000.

23. WU Q., MUELLER G.M., LUTZONI F.M., HUANG Y., GUO S. Phylogenetic and biogeographic relationships of eastern Asian and eastern North American disjunct Suillus species (fungi) as inferred from nuclear ribosomal RNA ITS sequences. Mol. Phylogenet. Evol. 17 (1), 37, 2000.

24. KIKUCHI K., MATSUSHITA N., GUERIN-LAGUETTE A., OHTA A., SUZUKI K. Detection of Tricholoma matsutake by specific ITS primers. Mycol. Res. 104 (104), 1427, 2001.

25. ZAJC J., LIU Y., DAI W., YANG Z., HU J., GOSTINČAR C. Genome and transcriptome sequencing of the halophilic fungus Wallemia ichthyophaga: haloadaptations present and absent. BMC Genomics. 14 (18), 617, 2013.

26. LEE S.S., HONG S.W., CHUNG H.C., SUNG C.K., KIM J.H., KA K.H., KIM H.J. The specific probes confirming the genomic DNA of Tricholoma matsutake in Korea. Korean. J. Mycol. 27 (1), 20, 1999.

27. YU M., YU J., GU C., NIE Y., CHEN Z., YIN X., LIU $\mathrm{Y}$. De novo sequencing and transcriptome analysis of Ustilaginoidea virens by using Illumina paired-end sequencing and development of simple sequence repeat markers. Gene. 547 (2), 202, 2014.

28. XIANG D., YILING H. Identification of Genetic characterization and volatile compounds of Tricholoma matsutake from different geographical origins, Biochem. Syst. Ecol. 44 (44), 233, 2012.

29. GARDES M., BRUNS T.D. ITS primers with enhanced specificity for basidiomycetes - application to the identification of mycorrhizae and rusts. Mol. Ecol. 2 (2), $113, \mathbf{1 9 9 3}$

30. AMICUCCI A., ZAMBONELLI A., GIOMARO G., POTENZAL., STOCCHIV. Identification of ectomycorrhizal fungi of the genus tuber by species-specific ITS primers. Mol. Ecol. 7 (3), 273, 1998. 